\title{
McGill
}

\section{Structural imaging biomarkers of Alzheimer's disease: predicting disease progression}

\author{
Simon F. Eskildse, Pierrick Coupé, Vladimir Fonov, Jens C. \\ Pruessner, D. Louis Collins for the Alzheimer's Disease \\ Neuroimaging Initiative
}

Neurobiology of Aging, Volume 36, Supplement 1, January 2015, pp. S23-S31

doi: 10.1016/j.neurobiolaging.2014.04.034

http://www.sciencedirect.com/science/article/pii/S019745801400548X

Copyright 2015 Elsevier Inc. This postprint provided under the terms of a Creative Commons Attribution-NonCommercial-NonDerivative licence. 
Title: Structural imaging biomarkers of Alzheimer's disease: predicting disease progression

Authors: Simon F. Eskildsen ${ }^{\mathrm{a},{ }^{*}}$, Pierrick Coupéb ${ }^{b}$, Vladimir Fonov ${ }^{c}$, Jens C. Pruessner ${ }^{d}$, D. Louis Collins ${ }^{c}$ for the Alzheimer's Disease Neuroimaging Initiative ${ }^{1}$

${ }^{a}$ Center of Functionally Integrative Neuroscience, Aarhus University, Aarhus, Denmark

${ }^{\mathrm{b}}$ Laboratoire Bordelais de Recherche en Informatique, Unité Mixte de Recherche CNRS (UMR 5800), Bordeaux, France

${ }^{\mathrm{c}}$ McConnell Brain Imaging Centre, Montreal Neurological Institute, McGill University, Montreal, Canada

${ }^{d}$ Departments of Psychiatry, Neurology and Neurosurgery, McGill University, Montreal, Canada

Word count: 5194 (excluding references, figures and tables)

3 tables and 5 figures

${ }^{*}$ Corresponding author:

Simon Fristed Eskildsen

Center of Functionally Integrative Neuroscience, Aarhus University

Nørrebrogade 44, byg. $10 \mathrm{G}$

DK-8000, Aarhus, Denmark

Telephone: $+45-7846-9939$, mobile: $+45-2210-1234$, fax: $+45-8949-4400$

Email: seskildsen@cfin.au.dk

1 Data used in the preparation of this article were obtained from the Alzheimer's Disease Neuroimaging Initiative (ADNI) database (www.loni.ucla.edu/ADNI). As such, the investigators within the ADNI contributed to the design and implementation of $A D N I$ and/or provided data but did not participate in analysis or writing of this report. A complete listing of ADNI investigators can be found at:

http://adni.loni.ucla.edu/wp-content/uploads/how_to_apply/ADNI_Authorship_List.pdf. 


\begin{abstract}
Optimized MRI-based biomarkers of Alzheimer's disease (AD) may allow earlier detection and refined prediction of the disease. In addition, they could serve as valuable tools when designing therapeutic studies of individuals at risk of AD. In this study we combine (i) a novel method for grading medial temporal lobe structures with (ii) robust cortical thickness measurements to predict $A D$ among subjects with mild cognitive impairment $(\mathrm{MCl})$ from a single T1-weighted MRI scan. Using $A D$ and cognitively normal individuals, we generate a set of features potentially discriminating between $\mathrm{MCl}$ subjects who convert to $\mathrm{AD}$ and those that remain stable over a period of three years. Using mutual information based feature selection we identify five key features optimizing the classification of $\mathrm{MCl}$ converters. These features are the left and right hippocampus grading and cortical thicknesses of the left precuneus, left superior temporal sulcus, and right anterior part of the parahippocampal gyrus. We show that these features are highly stable in cross validation and enable a prediction accuracy of $72 \%$ using a simple linear discriminant classifier; the highest prediction accuracy obtained on the baseline ADNI1 cohort to date. The proposed structural features are consistent with Braak stages and previously reported atrophic patterns in AD and are easy to transfer to new cohorts and to clinical practice.
\end{abstract}

Keywords: Alzheimer, MCI, MRI, early detection, prediction, SNIPE, FACE, hippocampus, cortical thickness 


\section{Introduction}

Neuronal injury is an integral part of the pathophysiological process of Alzheimer's disease (AD). Measures of neuronal injury and neurodegeneration are among the most important biomarkers of AD (Jack et al., 2012). Cerebral atrophy caused by the progressive neurodegeneration can be measured in detail by magnetic resonance imaging (MRI). Anatomical MRI is routinely carried out in clinical practice when diagnosing patients with cognitive disturbances, such as memory problems, to eliminate other possible symptom causes. Thus, atrophy biomarkers based on MRI have a minimal cost impact since MRI scanning is often part of the standard assessment. Optimizing such MRI-based biomarkers for detection and prediction of AD may have a significant impact on early diagnosis of patients as well as being valuable tools when designing therapeutic studies of individuals at risk of $A D$ to prevent or alter the progression of the disease.

Hippocampal atrophy has long been recognized as an early feature of the degenerative process in AD (Ball et al., 1985). Reductions in hippocampal volume appear to correspond to early memory decline (De Leon et al., 1989). While sensitive, hippocampal degeneration is involved in other dementias, such as vascular dementia (Gainotti et al., 2004), and is known to be part of non-pathological brain aging (Driscoll et al., 2003). Thus, volumetric measurements of the hippocampus $(\mathrm{HC})$ are limited in their ability to predict the progression of AD (Chupin et al., 2009; Clerx et al., 2013; Coupé et al., 2012; Wolz et al., 2011). Evidence suggests that the nature of degeneration in the $\mathrm{HC}$ and surrounding structures, such as the entorhinal cortex (ERC) and parahippocampal gyrus, is different in AD compared to other dementias and different from the changes occurring during normal aging (Devanand et al., 2012). We have recently obtained results that support this finding; prediction can be improved by considering the structural composition of the $\mathrm{HC}$ and its surrounding structures in the medial temporal lobe (Coupé et al., 2012). Our results were obtained using a novel concept of measuring structural similarities, comparing the anatomy of a test subject to a library of $A D$ patients and cognitive normal $(C N)$ subjects. 
Studies have shown that, apart from hippocampal and medial temporal lobe (MTL) atrophy, AD has a characteristic neocortical atrophy pattern (Dickerson et al., 2009; McEvoy et al., 2009). Cortical thinning of temporal and parietal lobe regions, the posterior cingulate and the precuneus seem to be involved at early stages of the disease (Reiman and Jagust, 2012). In the advanced stages of the disease, atrophy spreads to almost the entire cortex sparing only the sensory-motor and visual cortex (Eskildsen et al., 2012b). Recently, we showed in (Eskildsen et al., 2013) that if cortical thickness is measured in a consistent manner, patterns of cortical thinning can predict conversion to $A D$ among mild cognitive impaired $(\mathrm{MCl})$ subjects with higher accuracy (68\%) compared to conventional voxel based morphometry (56\%) (Davatzikos et al., 2011) and deformation based morphometry (64\%) (Wolz et al., 2011).

In the current study we combine measurements of structural pathological patterns, measured by analyzing morphological alterations, in key structures of the MTL with degenerative patterns of the neocortex, measured by cortical thickness, to determine if prediction accuracies can be improved further by considering the entire gray matter (GM) atrophy footprint of AD. Moreover, we study the advantage of using feature selection to extract the highest relevant information from a set of potential discriminant features.

\section{Methods}

\subsection{Participants and imaging}

Data used in the preparation of this article were obtained from the Alzheimer's Disease Neuroimaging Initiative (ADNI) database (adni.loni.ucla.edu). The primary goal of ADNI has been to test whether serial MRI, positron emission tomography (PET), other biological markers, and clinical and neuropsychological assessment can be combined to measure the progression of $\mathrm{MCl}$ and early $\mathrm{AD}$. Determination of sensitive and specific markers of very early AD progression is intended to aid researchers and clinicians to develop new treatments and monitor their effectiveness, as well as lessen the time and cost of clinical trials. ADNI began 
in 2004 and is ongoing, now in its third phase (ADNI2). In this study we focused on the now completed first phase of ADNI (ADNI1, 2004-2010). For up-to-date information, see www.adni-info.org.

In this study we selected all 834 ADNI1 subjects available at baseline or screening. Note that only 819 subjects were officially enrolled in ADNI1. However, in order to compare results with recently published studies on ADNI data (Coupé et al., 2012; Eskildsen et al., 2013; Liu et al., 2012; Wolz et al., 2011), we decided to conform to the subject inclusion criteria described in these papers. At baseline or screening 198 subjects were diagnosed as $A D$ patients, 405 subjects had $\mathrm{MCl}$, and 231 subjects were categorized as cognitively normal (CN). As done by Wolz et al. (2011), we determined progressive $\mathrm{MCl}(\mathrm{pMCl})$ as those patients who had a diagnosis of AD as of July 2011. The complementary group of $\mathrm{MCl}$ patients was considered stable $\mathrm{MCl}$ (sMCI). It should be noted that due to study drop-outs, and to the limited follow-up period, the label sMCI is uncertain for a potentially important group of $\mathrm{MCl}$ patients. Table 1 summarizes the cohorts in our study. For our analyses we used baseline (or screening) T1-weighted MRI acquired at 1.5T only. One of our goals was to determine how well we could predict conversion to AD using only cross-sectional data, as would be the case at the first visit of a patient in clinical practice. $A D$ and $C N$ subject baseline scans were used for extracting image features sensitive to the pathology, thus enabling an independent analysis for sMCl and $\mathrm{pMCl}$ populations. While there were no statistical significant differences in age or sex between AD and CN (age: $p=0.490$, sex: $p=0.652$ ) and between $s M C l$ and $p M C l$ (age: $p=0.532$, sex: $p=0.206$ ), it is important to note that there were statistically significant sex differences between the $\mathrm{MCl}$ cohort and the $\mathrm{AD}$ and $\mathrm{CN}$ cohorts. The $\mathrm{MCl}$ cohort has a significantly $(\mathrm{p}<0.005)$ lower rate of females. A complete list of the $\mathrm{MCl}$ subjects used in the analyses identified by ADNI ID can be found in the supplementary material of (Eskildsen et al., 2013).

\subsection{Image preprocessing}

All images were processed using a fully automatic pipeline (Aubert-Broche et al., 2013). Images were denoised (Coupe et al., 2008) using a Rician-adapted noise estimation (Coupe et al., 2010), bias field corrected 
(Sled et al., 1998), and registered to MNI space using a 12 parameter affine transformation (Collins et al., 1994). To enable robust registrations we used as registration target a population-specific template derived from the ADNI1 database constructed using a series of linear and non-linear registrations as described in (Fonov et al., 2011). The custom template was created from 50 AD patients and $50 \mathrm{CN}$ subjects randomly selected. This template better reflects the anatomy of ADNI data compared to the conventional ICBM template build from young healthy adults. Image intensities were normalized to match the intensity profile of the template (Nyul and Udupa, 2000), and finally the images were skull stripped using BEaST (Eskildsen et al., 2012a).

\subsection{Hippocampus and entorhinal cortex}

The SNIPE (Scoring by Nonlocal Image Patch Estimator) method was used to extract structural features of the hippocampal complex (Coupé et al., 2012). In this technique, the local structural information surrounding each voxel (i.e., 3D patch) of a test subject is compared to those in a library of pre-labeled MRI datasets from $A D$ and $C N$ subjects. In short, a small patch of MRI data around each voxel (e.g., $7 \times 7 \times 7$ voxel patches) from the test subject is compared to the training library in a non-local fashion, with a goal to find similar patches, where similar is defined by the sum of squared intensity differences (SSD). The patch similarity is used to compute a weight for the match and used to determine two pieces of information. First, the weight is integrated for the different voxel labels in the template library (i.e., background, HC or ERC) and the label with the maximum weight determines the label of the voxel in the test subject, thus achieving segmentation. Second, the weights are multiplied against the subject group label $(A D=-1.0, C N=+1.0)$ in the template library, and integrated over all matches, to determine if the voxel in question is more similar to the $A D$ group (with a negative integrated score) or the $\mathrm{CN}$ group (with a positive integrated score). More specific details are given in (Coupé et al., 2012). Before running SNIPE, images were cropped around the structures of interest and intensity cross-normalized between the subjects using a histogram based method applied within the MTL region (Nyul and Udupa, 2000). We used SNIPE to obtain both grading values and volumes of the left and right $\mathrm{HC}$ and ERC of all $\mathrm{MCl}$ subjects using the parameters proposed in (Coupé et al., 2012). 
As detailed in (Coupe et al., 2012), the first step to apply SNIPE to the ADNI dataset was to perform a label propagation. This step consisted in propagating a small number of manual segmentations over the entire training library using the segmentation described in (Coupe et al., 2011). As in (Coupe et al., 2012), we used 20 scans randomly selected from the $A D$ and $C N$ populations ( $10 \mathrm{CN}$ and $10 \mathrm{AD}$ ) for manual labeling by an expert using the protocol described in (Pruessner et al., 2002). Then, these manual segmentations were used to segment the entire $A D$ and $C N$ populations. Finally, $H C$ and ERC segmentations were available for the $231 \mathrm{CN}$ subjects and $198 \mathrm{AD}$ patients constituting our training library.

The second step of the SNIPE is the structure grading using automatic segmentations obtained from the propagation step. As proposed in (Coupé et al., 2012), the 50 closest subjects were first selected from each training population (i.e., $50 \mathrm{AD}$ and $50 \mathrm{CN}$ ) using SSD over an initialization mask. Then, the grading maps and the segmentations of the considered structure were obtained simultaneously using SNIPE. Finally, the 8 SNIPE-based features extracted were the average grading value over the left and right HC and ERC and the volume of the same four structures (feature 1-8). Volumes were calculated in normalized space (MNI space) to avoid bias toward head size.

\subsection{Neocortex}

Cortical thickness was calculated using FACE (Fast Accurate Cortex Extraction) (Eskildsen and Ostergaard, 2006; Eskildsen et al., 2005) and mapped to the cortical surface of the population-specific average nonlinear anatomical template (Fonov et al., 2011) using an iterative, feature-based algorithm (Eskildsen and Ostergaard, 2008). All $A D$ and $C N$ subjects were used to generate a statistical map of group differences in cortical thickness. From this t-map, cortical thickness features were derived with the procedure described in (Eskildsen et al., 2013) using the proportion of the cortical surface with the $20 \%$ largest t-values corresponding to a threshold of $\mathrm{t}=7.4$ (see Figure 2). In brief, candidate ROls were calculated using a multi-seed constrained surface based region growing algorithm initialized at local maxima of the $20 \% \mathrm{t}$-map. In 
(Eskildsen et al., 2013) we found a cortical area of 10-15\% to be suitable for searching for candidate ROIs. In the current study we decided to increase the threshold, and thereby the search area, to account for population differences between selection datasets and prediction datasets $(A D / C N$ vs. $\mathrm{pMCl} / \mathrm{sMCl})$. Note that here, as in the application of SNIPE above, the feature generation is completely independent of the test data. The $C N$ and $A D$ groups are used to determine the regions of interest (ROIs) in terms of cortical thickness, and these are used to evaluate the independent $\mathrm{MCl}$ group. $\mathrm{A}$ total of 63 candidate cortical thickness ROls were identified this way (see Figure 2). Neocortical features comprised the mean cortical thickness within each of these ROIs (feature 9-71) measured in subject native space.

\subsection{Quality control}

All results were visually evaluated for quality control by an expert and subjects were excluded if errors were found in one of the image processing steps mentioned above. Due to our highly robust image processing pipeline, only $3.1 \%$ of the images were excluded this way. The cohort numbers after quality control are given in Table 1. For the 24 datasets that failed, 5 were $\mathrm{CN}, 9$ were $\mathrm{sMCl}, 6$ were $\mathrm{pMCl}$ and 4 were $\mathrm{AD}$. Failures were due mostly to excessive motion artifacts or unexpected head position in the scanner. All other $\mathrm{CN}$ and $\mathrm{AD}$ data were used in the template library and all other $\mathrm{MCl}$ data were used in the experiments. Our $3.1 \%$ failure rate is much lower than the $12.8 \%$ failure rate reported by Wolz et al. (2011) on the same data for a similar analysis using cortical thickness.

\subsection{Feature selection}

Candidate features for the prediction of $A D$ among $M C l$ subjects included 71 image-based features (8 SNIPE features and 63 cortical thickness ROIs). To select discriminant features, we used a mutual information feature selection (MIFS) method (Battiti, 1994). In this method, features are selected iteratively in ascending order according to their mutual information score between feature and class and the already selected features. This way features are selected for being informative of the class without being predictable from the current set of features, thus removing redundancy in the feature set. In the given prediction problem, 
many of the candidate features can be assumed highly correlated, especially as cortical ROls are spatially close. Thus, we expect the MIFS algorithm to remove many redundant features while keeping the most informative ones. Feature values were standardized before calculating the mutual information. As the selection method does not provide any information of the optimal number of features, we automatically selected features using the MIFS method generating feature sets consisting of 1 to 30 features to be tested by the classifier. To get an unbiased estimate of the prediction accuracy, we carefully performed feature selection independent of the subject to be classified. This was done in a leave-one-out (LOO) fashion where the feature set optimizing the classification of the training set was used to classify the subject left out. This nested LOO procedure resulted in a specific feature set for each subject. To analyze the stability of the selected features we recorded how frequent each feature was chosen for optimal classification in the training data.

\subsection{Prediction}

Generated features were used for prediction of $\mathrm{MCl}$ subjects using a linear discriminant classifier (LDA) in the LOO cross validation procedure mentioned above. Eight different feature sets were tested: 1) Total MMSE score, 2) long delayed recall score of Rey auditory verbal learning test (RAVLT), 3), all cortical thickness features, 4) all SNIPE features, 5) all imaging features, 6) selected cortical thickness features, 7) selected SNIPE features, and 8) selected features among all imaging features (identified below as the combined feature set). This was done to evaluate the added prediction power of each method and the effect of using feature selection. Prediction using MMSE alone was chosen as a reference as this neuropsychological assessment is widely used in clinical practice for screening purposes, and permits comparisons with previous literature using the ADNI database. However, studies have shown that episodic memory may be a better predictor of disease progression (Balthazar et al., 2010; Mormino et al., 2009). Thus we included RAVLT scores for comparison in our analysis. To be of any value, predictions based on imaging should improve the prediction based on cognitive scores alone. In all scenarios the subject age was included as feature, as our 
previous results have shown improved prediction accuracies when age is used in combination with structural features (Coupe et al., 2012; Eskildsen et al., 2013).

\section{Results}

When using SNIPE features only, the feature selection method selected left and right HC grading values in all LOO experiments (Table 2). The next most selected features were only selected in about quarter of the experiments. In most experiments two features were found to be optimal. This fully automatic feature selection is in line with results obtained in (Coupé et al., 2012) doing an exhaustive search of the optimal combination of SNIPE features. Using cortical thickness features only, the left precuneus were chosen in all cases followed by the left parahippocampal gyrus and an area corresponding to the uncal recess of the inferior horn of the lateral ventricle on top of the hippocampus. In this case, three features were found to be optimal for most classification experiments. Interestingly, when applying the feature selection on the combined feature set, the stability of the selected features increased. The same five imaging features were selected in almost all experiments. These were left and right $\mathrm{HC}$ grading values and cortical thickness of the left precuneus, left superior temporal sulcus and right anterior part of parahippocampal gyrus. Figure 3 visualizes the cortical thickness features most frequently selected for cortical thickness only and the combined feature set, respectively. The same five features were chosen in almost all experiments, making five the optimal number of features. Figure 4 visualizes the estimated prediction accuracy using varying number of selected features of the combined feature set.

Table 3 lists the results of predicting conversion using the LDA classifier in the eight different scenarios. Four things should be noted: 1) using just MMSE and age do not provide better accuracy than random selection ( $p=0.07$, McNemar's test), while all other feature sets do $(p<0.05), 2)$ SNIPE features yield nominally higher accuracies than cortical thickness features, though differences were not significant $(p>0.05$, see below), 3) applying feature selection increases accuracy ( $p \leq 0.003$, see below), and 4$)$ the combined selected features reach the best prediction among the tested scenarios, though only borderline significantly better 
than SNIPE ( $p=0.068$, see below). Figure 5 shows the corresponding receiver operating characteristic (ROC) curves for the three scenarios with feature selection. Using DeLong's test for two ROC curves (DeLong et al., 1988) revealed that the area under the curve (AUC) for the combined classifier was significantly different from the AUC of the cortical thickness classifier $(p=0.027)$ and almost significantly different from the AUC of the SNIPE classifier $(p=0.068)$. AUCs were not significantly different between the SNIPE and the cortical thickness classifiers both with and without feature selection. Using feature selection significantly improved the AUC in all feature sets $(p \leq 0.003)$. Though prediction using RAVLT scores was significantly better than random selection $(p=0.028)$, RAVLT was not significantly better than MMSE $(p=0.31)$. The classifier using the combined feature set performed significantly better than RAVLT scores $(p=0.012)$.

\section{Discussion}

The results demonstrate that the optimal prediction accuracy can be obtained using a set of few selected features from the medial and lateral temporal lobes and the precuneus. Adding further features reduces the prediction accuracy as additional noise is introduced without adding discriminative information. In general, it seems that there are many irrelevant candidates within the cortical thickness features. This might result from that fact that the cortical thickness features are based on statistical differences between $A D$ patients and $\mathrm{CN}$ subjects. The same could be said for the SNIPE features as they too were built from AD/CN templates. However, in this case the features are predetermined and not driven by the AD/CN contrast. Even though the t-map of cortical $A D / C N$ differences covers the regions involved in early $A D$, there are also large parts of the cortex that are affected in the later stages of the disease. Therefore, candidate features not suited for the $\mathrm{sMCl} / \mathrm{pMCl}$ classification are also generated by the feature extraction method. However, the mutual information feature selection (MIFS) method (Battiti, 1994) manages to identify a few discriminative ROIs from the 63 candidate ROls. In the future, we will investigate feature selection based on the $\mathrm{sMCl} / \mathrm{pMCl}$ differences. 
From Table 2 it can be observed that the same five features are selected in almost all the LOO experiments of the combined feature set and that in Figure 4 these five features seem to optimize the prediction accuracy. This indicates that the method proposed to generate and select features is stable and should generalize well to other datasets. The stability of the estimated prediction accuracy (indicated by the small standard error in Figure 4) indicates that our reported prediction accuracy of $72 \%$ is what can be expected in a real clinical prospective setup.

The prediction accuracy we obtain for conversion from $\mathrm{MCl}$ to $\mathrm{AD}$ is nominally higher than most recently published results on ADNI data (Chincarini et al., 2011; Cho et al., 2012; Chupin et al., 2009; Coupé et al., 2012; Cuingnet et al., 2011; Davatzikos et al., 2011; Eskildsen et al., 2013; Gray et al., 2013; Westman et al., 2013; Westman et al., 2011; Wolz et al., 2011). Though cohort definitions and sizes vary between studies, our cohort definition is identical to several of these published works (Coupé et al., 2012; Eskildsen et al., 2013; Wolz et al., 2011). A few studies report slightly higher accuracy than we obtain here (Li et al., 2012; Misra et al., 2009; Wee et al., 2012). However, these studies are all based on a smaller subset of the cohorts studied here and thus comparability with the current results is limited. In addition, non-linear classifiers were applied in these studies, which can potentially lead to unstable feature selection during training (Kalousis et al., 2007). Here, we demonstrate relatively high accuracy on all baseline ADNI1 data (excluding the $3 \%$ failing the image processing) using a linear classifier and few features, which are highly stable when cross-validated, imposing more confidence in the selected features.

The improvement in prediction accuracy over similar methods based on T1w MRI (Chincarini et al., 2011; Cho et al., 2012; Chupin et al., 2009; Cuingnet et al., 2011; Westman et al., 2013; Wolz et al., 2011) as demonstrated here is likely due to 1 ) the robustness and consistency of the image processing pipeline demonstrated by the very low failure rate, 2 ) the complementarity of the features extracted from the images (MTL and neocortical structures), as the neurodegenerative process in AD occurs across several regions of the brain, 3) the use of a robust and consistent feature extraction method reducing noise in the 
measurements, and 4) the high relevance of certain features such as $\mathrm{HC}$ grading and thickness of left precuneus and left superior temporal sulcus. The selection strategy showed that only a few key features extracted from structural MRI are sufficient for reaching prediction accuracy superior to state-of-the-art methods involving several thousands of features (Chincarini et al., 2011) or involving multi-modal features including MRI and PET (Gray et al., 2013).

Studies to date suggest a natural limit in the prediction accuracy obtained on the ADNI data. No method achieves higher accuracy than we report here on the baseline ADNI1 cohort, thus one can assume an upper limit of the prediction accuracy well under $100 \%$. This limitation may have several explanations. First, due to study drop-outs and limited follow-up, the status of a potentially important group of $\mathrm{MCl}$ subjects remain unknown, yet they are all grouped together as $\mathrm{sMCl}$. Second, many $\mathrm{MCl}$ subjects may have mixed pathologies affecting their cognitive performance. Thus, subjects not converting to AD may still suffer from other neurodegenerative processes with similar or overlapping atrophy patterns compared to the atrophy pattern of $A D$. Third, subjects diagnosed as $S M C I$ might still convert to $A D$ in the future, but since they have not done so yet are misdiagnosed as $\mathrm{sMCl}$ and confound the analysis. Finally, there is still a high uncertainty attached to the clinical diagnosis of AD. All of these issues will negatively affect the training of a classifier and impair its predictive power.

Contrary to recently reported prediction results on the ADNI data that rely on complex combinations of features (Chincarini et al., 2011; Cho et al., 2012; Li et al., 2012; Wee et al., 2012), we wanted to have simple, interpretable features. This was obtained by selecting a set of features linked to specific anatomical structures (not voxel or vertex level) and not transforming the feature space as done in many feature reduction methods (Brunzell and Eriksson, 2000). With a small feature set as we report here it is easier to replicate findings on other cohorts. In addition, by doing feature selection instead of feature reduction, new knowledge can be put into the context of the pathophysiological process of AD. 
The stability of which the same set of features were selected in the LOO experiments show that these features are measuring key processes involved in early AD. Contrary to other methods (Misra et al., 2009; Wee et al., 2012; Westman et al., 2013), the features proposed here do not need any normalization or modulation, are stable across sites with well-aligned scanner parameters (e.g., 59 different acquisition sites are involved in ADNI), and do not rely on longitudinal information as they can be obtained from a single T1weighted MRI sequence. It is very important to be able to predict outcome already at patient's first visit. Waiting for example 6 to 12 months for a follow-up scan may unnecessarily delay initiation of crucial neuroprotective therapies.

The regions we report to be the most sensitive in predicting $A D$ among subjects with $M C l$ overlaps with atrophic regions identified in early AD (Apostolova and Thompson, 2008; Desikan et al., 2010; Reiman and Jagust, 2012; Vemuri et al., 2008) and are consistent with the Braak stages (Braak and Braak, 1991). The selection of the left precuneus as a predictive feature for the disease progression is notable. Ishii et al. (2005) showed that GM density of the precuneus was lower in early onset $A D$ (EOAD) compared to late onset $A D$ (LOAD), while there were no statistical differences in GM densities in the MTL between groups. EOAD has a more aggressive course and higher pathological burden than LOAD. Even though none of the subjects enrolled in our study can be characterized as EOAD, we speculate that atrophy of the precuneus may be a sign that the pathology is taking a more progressive trajectory than those with mainly MTL atrophy. It should be noted that the features selected to optimize the prediction of $A D$ do not reflect the full atrophic pattern of early structural changes. Several potentially pathologically important ROls correlated with selected regions are excluded by the classifier due to information redundancy.

The grading method obtained by SNIPE, though quantified over a single structure, includes information of the surrounding structures. Indeed, while $\mathrm{HC}$ grading value is estimated over the $\mathrm{HC}$ segmentation only, the involved patch similarity is estimated between patches of $7 \times 7 \times 7$ voxels $(\mathrm{mm})$ and thus includes larger structural patterns. Therefore, the $\mathrm{HC}$ grading may encode pathological structures found in the parahippocam- 
pal gyrus and other neighboring anatomy. This is further supported by the fact that the feature selection method chose cortical ROls away from the $\mathrm{HC}$ region in the combined feature set, where both the left and right $\mathrm{HC}$ grading were chosen in all experiments. Our results demonstrate that grading values of the $\mathrm{HC}$ are compact measures of MTL changes due to disease and captures the early neurodegenerative process in AD yielding relatively high prediction accuracy. This enables even higher prediction accuracy with the support of only a few key cortical ROIs.

In line with previous work (Balthazar et al., 2010; Mormino et al., 2009), we find that episodic memory seems to be a better predictor of disease progression than global cognitive scores, such as MMSE. However, our results demonstrate that a small set of features extracted from structural MRI significantly improve prediction over neuropsychological tests.

\section{Conclusion}

We demonstrate that $A D$ can be predicted with relatively high accuracy using a small set of key anatomical features extracted from a single T1-weighted MR image. The prediction accuracy of $72 \%$ is the highest reported on the entire baseline ADNI1 cohort. The improvement in accuracy is likely due to the application of a novel and compact description of structural changes in the medial temporal lobe combined with neocortical thickness features and a highly robust image processing pipeline. Using feature selection based on mutual information lead to the selection of only five features highly stable under cross validation. The five proposed features are the left and right HC grading and the cortical thickness of the left precuneus, left superior temporal sulcus, and right anterior part of the parahippocampal gyrus. These are consistent with Braak stages and previously reported atrophic patterns in AD and are easy to transfer to new cohorts and to clinical practice. 


\section{Acknowledgements}

This work was funded in part by operating grants from the Canadian Institutes of Health Research, les Fonds de la recherche santé du Quebec, MINDLab UNIK initiative at Aarhus University, funded by the Danish Ministry of Science, Technology and Innovation, grant agreement number 09-065250. Data collection and sharing for this project were funded by the Alzheimer's Disease Neuroimaging Initiative (ADNI) (National Institutes of Health Grant U01 AG024904). The ADNI is funded by the National Institute on Aging and the National Institute of Biomedical Imaging and Bioengineering and through generous contributions from the following: Abbott, AstraZeneca AB, Bayer Schering Pharma AG, Bristol-Myers Squibb, Eisai Global Clinical Development, Elan Corporation, Genentech, GE Healthcare, GlaxoSmithKline, Innogenetics NV, Johnson \& Johnson, Eli Lilly and Co., Medpace, Inc., Merck and Co., Inc., Novartis AG, Pfizer Inc., F. HoffmannLa Roche, Schering-Plough, Synarc Inc., as well as nonprofit partners, the Alzheimer's Association and Alzheimer's Drug Discovery Foundation, with participation from the U.S. Food and Drug Administration. Private sector contributions to the ADNI are facilitated by the Foundation for the National Institutes of Health (www.fnih.org). The grantee organization is the Northern California Institute for Research and Education, and the study was coordinated by the Alzheimer's Disease Cooperative Study at the University of California, San Diego. ADNI data are disseminated by the Laboratory for Neuro Imaging at the University of California, Los Angeles. This research was also supported by NIH grants P30AG010129, K01 AG030514 and the Dana Foundation.

\section{Disclosure statement}

FACE is the subject of European Patent No. EP04011386.2. and US Patent No. 060/570,493. SNIPE is the subject of a US provisional patent application (US 61/535,720 / P1310USPR). D.L. Collins is a consultant for NeuroRx Inc. and co-founder of True Positive Medical Devices Inc. 


\section{References}

Apostolova, L.G., Thompson, P.M., 2008. Mapping progressive brain structural changes in early Alzheimer's disease and mild cognitive impairment. Neuropsychologia 46, 1597-1612.

Aubert-Broche, B., Fonov, V.S., Garcia-Lorenzo, D., Mouiha, A., Guizard, N., Coupe, P., Eskildsen, S.F., Collins, D.L., 2013. A new method for structural volume analysis of longitudinal brain MRI data and its application in studying the growth trajectories of anatomical brain structures in childhood. Neurolmage 82C, 393-402.

Ball, M.J., Fisman, M., Hachinski, V., Blume, W., Fox, A., Kral, V.A., Kirshen, A.J., Fox, H., Merskey, H., 1985. A new definition of Alzheimer's disease: a hippocampal dementia. Lancet 1, 14-16.

Balthazar, M.L., Yasuda, C.L., Cendes, F., Damasceno, B.P., 2010. Learning, retrieval, and recognition are compromised in aMCl and mild $\mathrm{AD}$ : are distinct episodic memory processes mediated by the same anatomical structures? Journal of the International Neuropsychological Society : JINS 16, 205-209.

Battiti, R., 1994. Using mutual information for selecting features in supervised neural net learning. Neural Networks, IEEE Transactions on 5, 537-550.

Brunzell, H., Eriksson, J., 2000. Feature reduction for classification of multidimensional data. Pattern Recognition 33, 1741-1748.

Braak, H., Braak, E., 1991. Neuropathological stageing of Alzheimer-related changes. Acta Neuropathol 82, 239-259.

Chincarini, A., Bosco, P., Calvini, P., Gemme, G., Esposito, M., Olivieri, C., Rei, L., Squarcia, S., Rodriguez, G., Bellotti, R., Cerello, P., De Mitri, I., Retico, A., Nobili, F., 2011. Local MRI analysis approach in the diagnosis of early and prodromal Alzheimer's disease. Neurolmage 58, 469-480.

Cho, Y., Seong, J.K., Jeong, Y., Shin, S.Y., 2012. Individual subject classification for Alzheimer's disease based on incremental learning using a spatial frequency representation of cortical thickness data. Neurolmage 59, 2217-2230.

Chupin, M., Gerardin, E., Cuingnet, R., Boutet, C., Lemieux, L., Lehericy, S., Benali, H., Garnero, L., Colliot, O., 2009. Fully automatic hippocampus segmentation and classification in Alzheimer's disease and mild cognitive impairment applied on data from ADNI. Hippocampus 19, 579-587.

Clerx, L., van Rossum, I.A., Burns, L., Knol, D.L., Scheltens, P., Verhey, F., Aalten, P., Lapuerta, P., van de Pol, L., van Schijndel, R., de Jong, R., Barkhof, F., Wolz, R., Rueckert, D., Bocchetta, M., Tsolaki, M., Nobili, F., Wahlund, L.-O., Minthon, L., Frölich, L., Hampel, H., Soininen, H., Visser, P.J., 2013. Measurements of medial temporal lobe atrophy for prediction of Alzheimer's disease in subjects with mild cognitive impairment. Neurobiology of aging.

Collins, D.L., Neelin, P., Peters, T.M., Evans, A.C., 1994. Automatic 3D intersubject registration of MR volumetric data in standardized Talairach space. Journal of computer assisted tomography 18, 192-205.

Coupe, P., Manjon, J.V., Fonov, V., Pruessner, J., Robles, M., Collins, D.L., 2011. Patch-based segmentation using expert priors: application to hippocampus and ventricle segmentation. Neurolmage 54, 940-954.

Coupe, P., Eskildsen, S.F., Manjon, J.V., Fonov, V.S., Collins, D.L., 2012. Simultaneous segmentation and grading of anatomical structures for patient's classification: application to Alzheimer's disease. Neurolmage 59, 3736-3747. 
Coupé, P., Eskildsen, S.F., Manjón, J.V., Fonov, V.S., Pruessner, J.C., Allard, M., Collins, D.L., 2012. Scoring by nonlocal image patch estimator for early detection of Alzheimer's disease. Neurolmage: Clinical 1, 141-152.

Coupe, P., Manjon, J.V., Gedamu, E., Arnold, D., Robles, M., Collins, D.L., 2010. Robust Rician noise estimation for MR images. Med Image Anal 14, 483-493.

Coupe, P., Yger, P., Prima, S., Hellier, P., Kervrann, C., Barillot, C., 2008. An optimized blockwise nonlocal means denoising filter for 3-D magnetic resonance images. IEEE transactions on medical imaging 27, 425441.

Cuingnet, R., Gerardin, E., Tessieras, J., Auzias, G., Lehericy, S., Habert, M.O., Chupin, M., Benali, H., Colliot, O., 2011. Automatic classification of patients with Alzheimer's disease from structural MRI: a comparison of ten methods using the ADNI database. Neurolmage 56, 766-781.

Davatzikos, C., Bhatt, P., Shaw, L.M., Batmanghelich, K.N., Trojanowski, J.Q., 2011. Prediction of MCI to AD conversion, via MRI, CSF biomarkers, and pattern classification. Neurobiology of aging 32, 2322 e23192327.

De Leon, M., George, A., Stylopoulos, L., Smith, G., Miller, D., 1989. EARLY MARKER FOR ALZHEIMER'S DISEASE: THE ATROPHIC HIPPOCAMPUS. The Lancet 334, 672-673.

DeLong, E.R., DeLong, D.M., Clarke-Pearson, D.L., 1988. Comparing the areas under two or more correlated receiver operating characteristic curves: a nonparametric approach. Biometrics 44, 837-845.

Desikan, R.S., Cabral, H.J., Settecase, F., Hess, C.P., Dillon, W.P., Glastonbury, C.M., Weiner, M.W., Schmansky, N.J., Salat, D.H., Fischl, B., 2010. Automated MRI measures predict progression to Alzheimer's disease. Neurobiology of aging 31, 1364-1374.

Devanand, D.P., Bansal, R., Liu, J., Hao, X., Pradhaban, G., Peterson, B.S., 2012. MRI hippocampal and entorhinal cortex mapping in predicting conversion to Alzheimer's disease. Neurolmage 60, 1622-1629.

Dickerson, B.C., Bakkour, A., Salat, D.H., Feczko, E., Pacheco, J., Greve, D.N., Grodstein, F., Wright, C.I., Blacker, D., Rosas, H.D., Sperling, R.A., Atri, A., Growdon, J.H., Hyman, B.T., Morris, J.C., Fischl, B., Buckner, R.L., 2009. The Cortical Signature of Alzheimer's Disease: Regionally Specific Cortical Thinning Relates to Symptom Severity in Very Mild to Mild AD Dementia and is Detectable in Asymptomatic Amyloid-Positive Individuals. Cerebral cortex 19, 497-510.

Driscoll, I., Hamilton, D.A., Petropoulos, H., Yeo, R.A., Brooks, W.M., Baumgartner, R.N., Sutherland, R.J., 2003. The aging hippocampus: cognitive, biochemical and structural findings. Cerebral cortex $13,1344-$ 1351.

Eskildsen, S.F., Coupe, P., Fonov, V., Manjon, J.V., Leung, K.K., Guizard, N., Wassef, S.N., Ostergaard, L.R., Collins, D.L., 2012a. BEaST: brain extraction based on nonlocal segmentation technique. Neurolmage 59, 2362-2373.

Eskildsen, S.F., Coupe, P., Garcia-Lorenzo, D., Fonov, V., Pruessner, J.C., Collins, D.L., 2013. Prediction of Alzheimer's disease in subjects with mild cognitive impairment from the ADNI cohort using patterns of cortical thinning. Neurolmage 65C, 511-521.

Eskildsen, S.F., Fonov, V., Coupe, P., Collins, D.L., 2012b. Visualizing stages of cortical atrophy in progressive $\mathrm{MCl}$ from the ADNI cohort. Alzheimer's Association International Conference on Alzheimer's Disease. Alzheimer's Dementia, Vancouver, Canada. 
Eskildsen, S.F., Uldahl, M., Ostergaard, L.R., 2005. Extraction of the cerebral cortical boundaries from MRI for measurement of cortical thickness. pp. 1400-1410.

Eskildsen, S.F., Ostergaard, L.R., 2006. Active surface approach for extraction of the human cerebral cortex from MRI. Medical image computing and computer-assisted intervention: MICCAI ... International Conference on Medical Image Computing and Computer-Assisted Intervention 9, 823-830.

Eskildsen, S.F., Ostergaard, L.R., 2008. Evaluation of Five Algorithms for Mapping Brain Cortical Surfaces. Proceedings of the 2008 XXI Brazilian Symposium on Computer Graphics and Image Processing. IEEE Computer Society, pp. 137-144.

Fonov, V., Evans, A.C., Botteron, K., Almli, C.R., McKinstry, R.C., Collins, D.L., 2011. Unbiased average ageappropriate atlases for pediatric studies. Neurolmage 54, 313-327.

Gainotti, G., Acciarri, A., Bizzarro, A., Marra, C., Masullo, C., Misciagna, S., Tartaglione, T., Valenza, A., Colosimo, C., 2004. The role of brain infarcts and hippocampal atrophy in subcortical ischaemic vascular dementia. Neurol Sci 25, 192-197.

Gray, K.R., Aljabar, P., Heckemann, R.A., Hammers, A., Rueckert, D., 2013. Random forest-based similarity measures for multi-modal classification of Alzheimer's disease. Neurolmage 65, 167-175.

Ishii, K., Kawachi, T., Sasaki, H., Kono, A.K., Fukuda, T., Kojima, Y., Mori, E., 2005. Voxel-based morphometric comparison between early- and late-onset mild Alzheimer's disease and assessment of diagnostic performance of z score images. AJNR Am J Neuroradiol 26, 333-340.

Jack, C.R., Jr., Vemuri, P., Wiste, H.J., Weigand, S.D., Lesnick, T.G., Lowe, V., Kantarci, K., Bernstein, M.A., Senjem, M.L., Gunter, J.L., Boeve, B.F., Trojanowski, J.Q., Shaw, L.M., Aisen, P.S., Weiner, M.W., Petersen, R.C., Knopman, D.S., 2012. Shapes of the trajectories of 5 major biomarkers of Alzheimer disease. Arch Neurol 69, 856-867.

Kalousis, A., Prados, J., Hilario, M., 2007. Stability of feature selection algorithms: a study on highdimensional spaces. Knowl. Inf. Syst. 12, 95-116.

Li, Y., Wang, Y., Wu, G., Shi, F., Zhou, L., Lin, W., Shen, D., 2012. Discriminant analysis of longitudinal cortical thickness changes in Alzheimer's disease using dynamic and network features. Neurobiology of aging 33, 427 e415-430.

Liu, M., Zhang, D., Shen, D., 2012. Ensemble sparse classification of Alzheimer's disease. Neurolmage 60, 1106-1116.

McEvoy, L.K., Fennema-Notestine, C., Roddey, J.C., Hagler, D.J., Holland, D., Karow, D.S., Pung, C.J., Brewer, J.B., Dale, A.M., 2009. Alzheimer Disease: Quantitative Structural Neuroimaging for Detection and Prediction of Clinical and Structural Changes in Mild Cognitive Impairment1. Radiology 251, 195-205.

Misra, C., Fan, Y., Davatzikos, C., 2009. Baseline and longitudinal patterns of brain atrophy in $\mathrm{MCl}$ patients, and their use in prediction of short-term conversion to AD: results from ADNI. Neurolmage 44, 1415-1422.

Mormino, E.C., Kluth, J.T., Madison, C.M., Rabinovici, G.D., Baker, S.L., Miller, B.L., Koeppe, R.A., Mathis, C.A., Weiner, M.W., Jagust, W.J., 2009. Episodic memory loss is related to hippocampal-mediated betaamyloid deposition in elderly subjects. Brain : a journal of neurology 132, 1310-1323.

Nyul, L.G., Udupa, J.K., 2000. Standardizing the MR image intensity scales: making MR intensities have tissue-specific meaning. In: Mun, S.K. (Ed.). SPIE, San Diego, CA, USA, pp. 496-504. 
Pruessner, J.C., Kohler, S., Crane, J., Pruessner, M., Lord, C., Byrne, A., Kabani, N., Collins, D.L., Evans, A.C., 2002. Volumetry of temporopolar, perirhinal, entorhinal and parahippocampal cortex from high-resolution MR images: considering the variability of the collateral sulcus. Cereb Cortex 12, 1342-1353.

Reiman, E.M., Jagust, W.J., 2012. Brain imaging in the study of Alzheimer's disease. Neurolmage 61, 505516.

Robin, X., Turck, N., Hainard, A., Tiberti, N., Lisacek, F., Sanchez, J.C., Muller, M., 2011. pROC: an opensource package for $\mathrm{R}$ and $\mathrm{S}+$ to analyze and compare ROC curves. BMC bioinformatics 12, 77.

Sled, J.G., Zijdenbos, A.P., Evans, A.C., 1998. A nonparametric method for automatic correction of intensity nonuniformity in MRI data. IEEE transactions on medical imaging 17, 87-97.

Vemuri, P., Whitwell, J.L., Kantarci, K., Josephs, K.A., Parisi, J.E., Shiung, M.S., Knopman, D.S., Boeve, B.F., Petersen, R.C., Dickson, D.W., Jack, C.R., Jr., 2008. Antemortem MRI based STructural Abnormality iNDex (STAND)-scores correlate with postmortem Braak neurofibrillary tangle stage. Neurolmage 42, 559-567.

Wee, C.Y., Yap, P.T., Shen, D., 2012. Prediction of Alzheimer's disease and mild cognitive impairment using cortical morphological patterns. Human brain mapping.

Westman, E., Aguilar, C., Muehlboeck, J.S., Simmons, A., 2013. Regional magnetic resonance imaging measures for multivariate analysis in Alzheimer's disease and mild cognitive impairment. Brain Topogr 26, 9-23.

Westman, E., Simmons, A., Muehlboeck, J.S., Mecocci, P., Vellas, B., Tsolaki, M., Kloszewska, I., Soininen, H., Weiner, M.W., Lovestone, S., Spenger, C., Wahlund, L.O., 2011. AddNeuroMed and ADNI: similar patterns of Alzheimer's atrophy and automated MRI classification accuracy in Europe and North America. Neurolmage $58,818-828$.

Wolz, R., Julkunen, V., Koikkalainen, J., Niskanen, E., Zhang, D.P., Rueckert, D., Soininen, H., Lotjonen, J., 2011. Multi-method analysis of MRI images in early diagnostics of Alzheimer's disease. PloS one 6, e25446. 
Table 1. Cohort sizes and baseline demographics for subjects used in the study.

\begin{tabular}{|l|c|c|c|c|c|}
\hline Cohort & $\begin{array}{l}\text { ADNI1 baseline } \\
\text { subjects }\end{array}$ & $\begin{array}{l}\text { Passed imaging quality } \\
\text { control }\end{array}$ & \% females & Age (mean \pm sd) & MMSE (mean \pm sd) \\
\hline $\mathrm{AD}$ & 198 & 194 & $50 \%$ & $75.3 \pm 7.5$ & $23.2 \pm 2.0$ \\
\hline $\mathrm{CN}$ & 231 & 226 & $48 \%$ & $76.0 \pm 5.0$ & $29.1 \pm 1.0$ \\
\hline $\mathrm{sMCl}$ & 238 & 227 & $33 \%$ & $74.9 \pm 7.7$ & $27.3 \pm 1.9$ \\
\hline $\mathrm{pMCl}$ & 167 & 161 & $40 \%$ & $74.5 \pm 7.2$ & $26.4 \pm 2.0$ \\
\hline
\end{tabular}

Table 2. Rate of feature involvement in the LOO experiments. Features not selected in any experiment are not shown in the table.

\begin{tabular}{|c|l|c|c|c|}
\hline \multirow{2}{*}{$\#$} & Feature location & \multicolumn{3}{|c|}{ Involved in LOO experiments } \\
\cline { 3 - 5 } & & SNIPE only & Thickness only & Combined \\
\hline 1 & Left HC grading & $100 \%$ & NA & $100 \%$ \\
\hline 2 & Right HC grading & $100 \%$ & NA & $100 \%$ \\
\hline 4 & Right ERC grading & $24 \%$ & NA & $0 \%$ \\
\hline 8 & Right ERC volume & NA & $0 \%$ \\
\hline 3 & Left ERC grading & $1 \%$ & NA & $0 \%$ \\
\hline 6 & Right HC volume & NA & $100 \%$ & $100 \%$ \\
\hline 30 & Left precuneus & NA & $0 \%$ & $100 \%$ \\
\hline 36 & Left superior temporal sulcus & NA & $0 \%$ & $92 \%$ \\
\hline 50 & Right parahippocampal gyrus & NA & $0 \%$ & $13 \%$ \\
\hline 61 & Right precuneus & NA & $0 \%$ & $5 \%$ \\
\hline 40 & Left superior temporal sulcus & NA & $0 \%$ & $4 \%$ \\
\hline 27 & Left superior temporal sulcus & NA & $0 \%$ & $4 \%$ \\
\hline 65 & Right superior temporal sulcus & NA & $0 \%$ & $4 \%$ \\
\hline 70 & Right superior temporal sulcus & NA & $0 \%$ & $3 \%$ \\
\hline 42 & Left superior temporal sulcus & NA & $1 \%$ & $2 \%$ \\
\hline 58 & Right fusiform gurys & NA & $0 \%$ & $1 \%$ \\
\hline 14 & Left fusiform gyrus & NA & $95 \%$ & $0 \%$ \\
\hline 25 & Left parahippocampal gyrus & NA & $87 \%$ & $0 \%$ \\
\hline 68 & Right uncal recess inferior horn & NA & $20 \%$ & $0 \%$ \\
\hline 45 & Right supramarginal gyrus & NA & $10 \%$ & $0 \%$ \\
\hline 62 & Right inferior temporal gyrus & NA & $8 \%$ & $0 \%$ \\
\hline 20 & Left superior frontal sulcus & NA & $4 \%$ & $0 \%$ \\
\hline 37 & Left parahippocampal gyrus & NA & $3 \%$ & $0 \%$ \\
\hline 57 & Right fusiform gyrus & $2 \%$ & $0 \%$ \\
\hline 31 & Left parahippocampal gyrus & NA & $0 \%$ \\
\hline 46 & Right middle frontal gyrus & & $0 \%$ \\
\hline 44 & Right superior frontal gyrus & NA & 0 \\
\hline
\end{tabular}


Table 3. Prediction results for the LDA classifier using various feature sets. AUC=Area under the receiver operating characteristic curve. MMSE=Mini-mental state examination. RAVLT=Rey auditory verbal learning test.

\begin{tabular}{|l|l|c|c|c|c|}
\hline Method & Feature set* & Accuracy & Sensitivity & Specificity & AUC \\
\hline MMSE & Total MMSE score, age & $59.3 \%$ & $56.5 \%$ & $61.2 \%$ & $62.9 \%$ \\
\hline RAVLT & Delayed recall score, age & $61.2 \%$ & $75.8 \%$ & $50.5 \%$ & $67.0 \%$ \\
\hline Cortical thickness, all & 63 ROIs, age & $63.9 \%$ & $60.9 \%$ & $66.1 \%$ & $66.4 \%$ \\
\hline SNIPE, all & 4 gradings, 4 volumes, age & $68.8 \%$ & $67.7 \%$ & $69.6 \%$ & $71.5 \%$ \\
\hline Combined, all & 71 image features, age & $67.3 \%$ & $64.6 \%$ & $69.2 \%$ & $70.1 \%$ \\
\hline Cortical thickness, selected & 3 ROls, age & $67.8 \%$ & $65.8 \%$ & $69.2 \%$ & $72.8 \%$ \\
\hline SNIPE, selected & L/R HC grading, age & $69.6 \%$ & $67.7 \%$ & $70.9 \%$ & $73.3 \%$ \\
\hline Combined, selected & L/R HC grading, 3 ROIs, age & $71.9 \%$ & $69.6 \%$ & $73.6 \%$ & $76.3 \%$ \\
\hline
\end{tabular}

* For the selected feature sets, the most frequent selected features are listed.

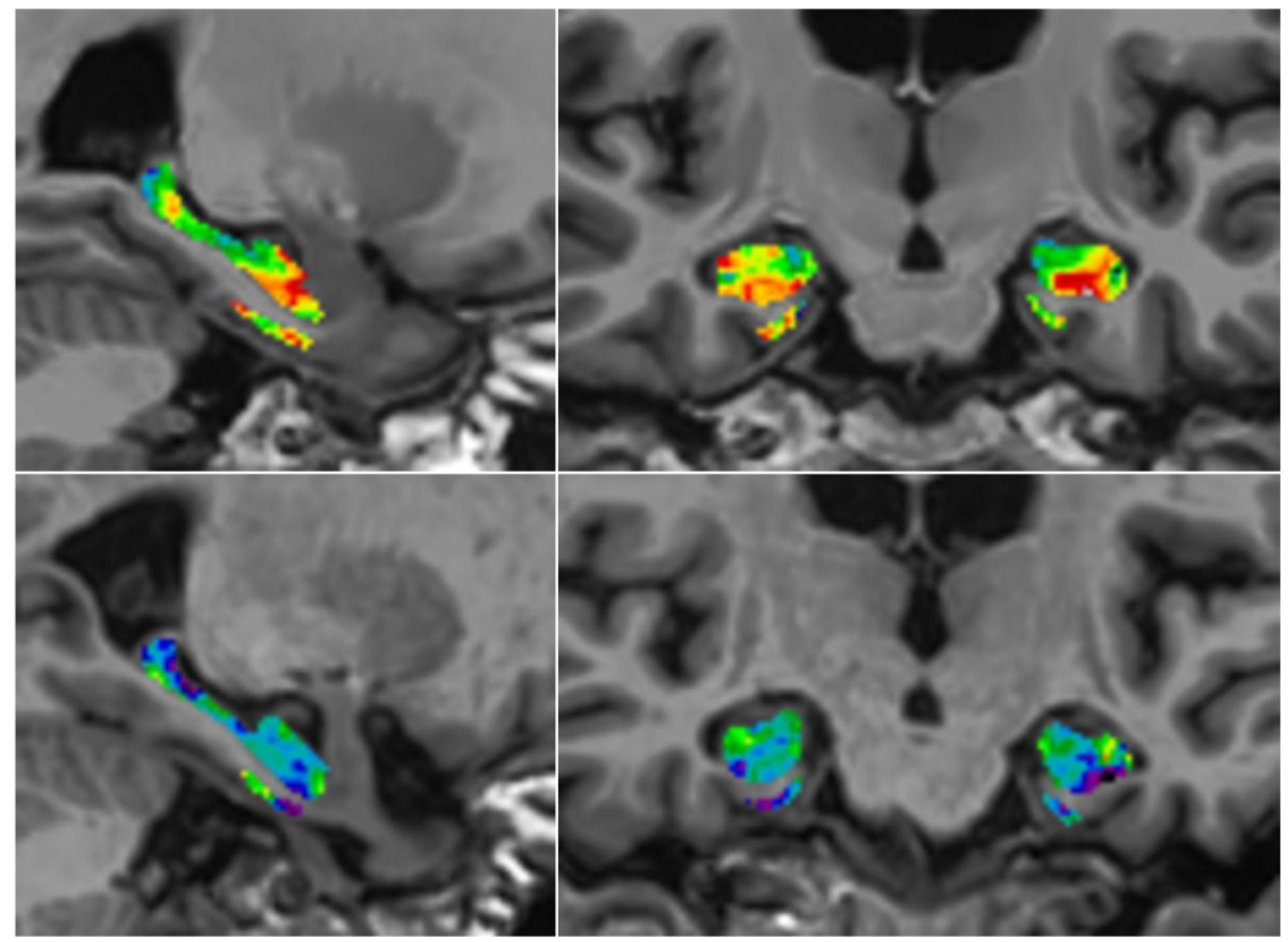

\section{Healthy}

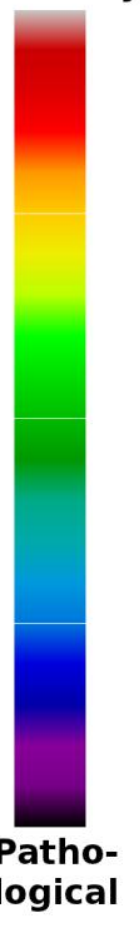

Figure 1. SNIPE grading of a sMCI subject (top row) and a pMCI subject (bottom row). 

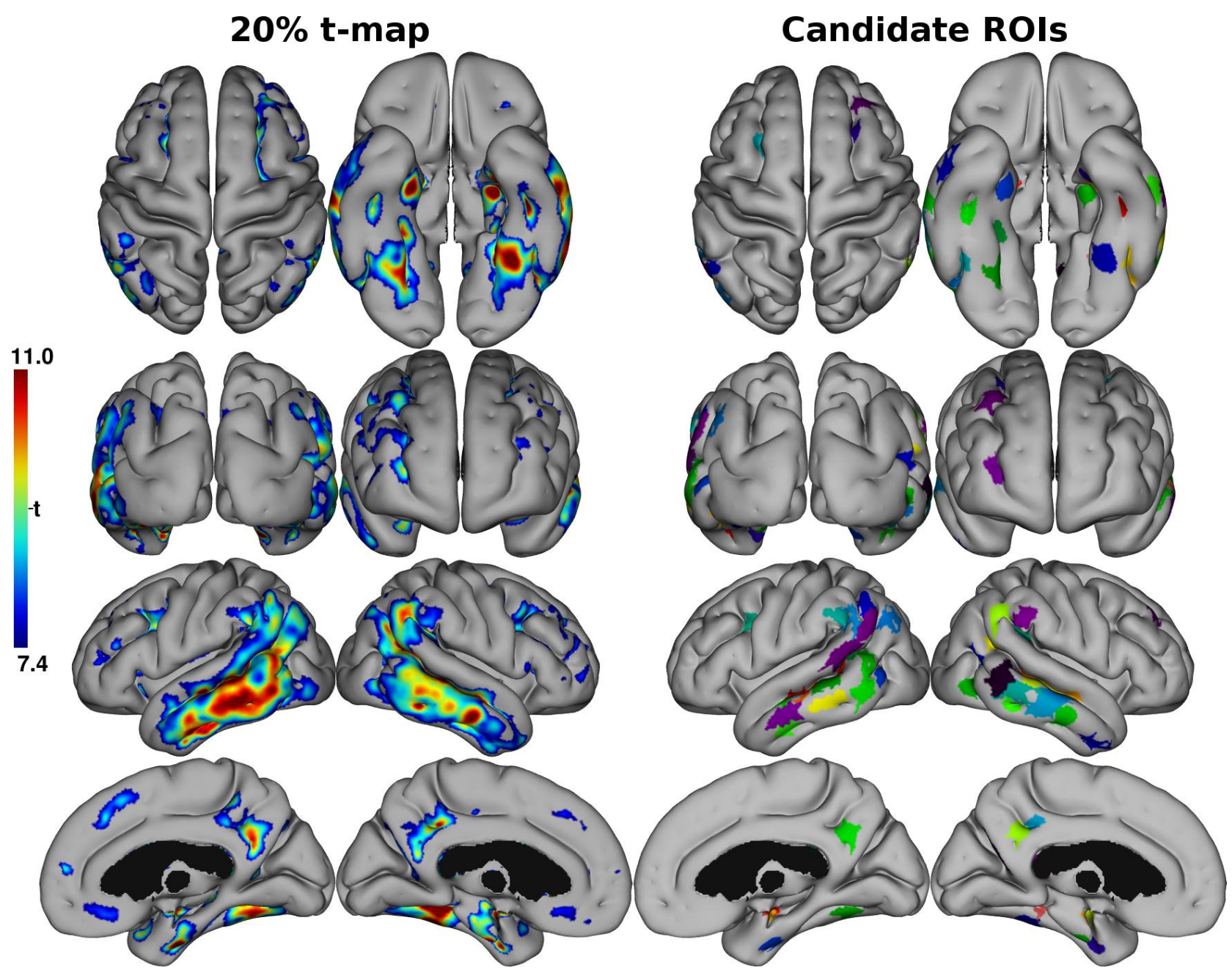

Figure 2. Feature selection based on cortical thickness AD vs. CN group differences. Left: Thresholded t-map showing the $20 \%$ largest t-values. Right: 63 ROIs automatically generated from the thresholded t-map.
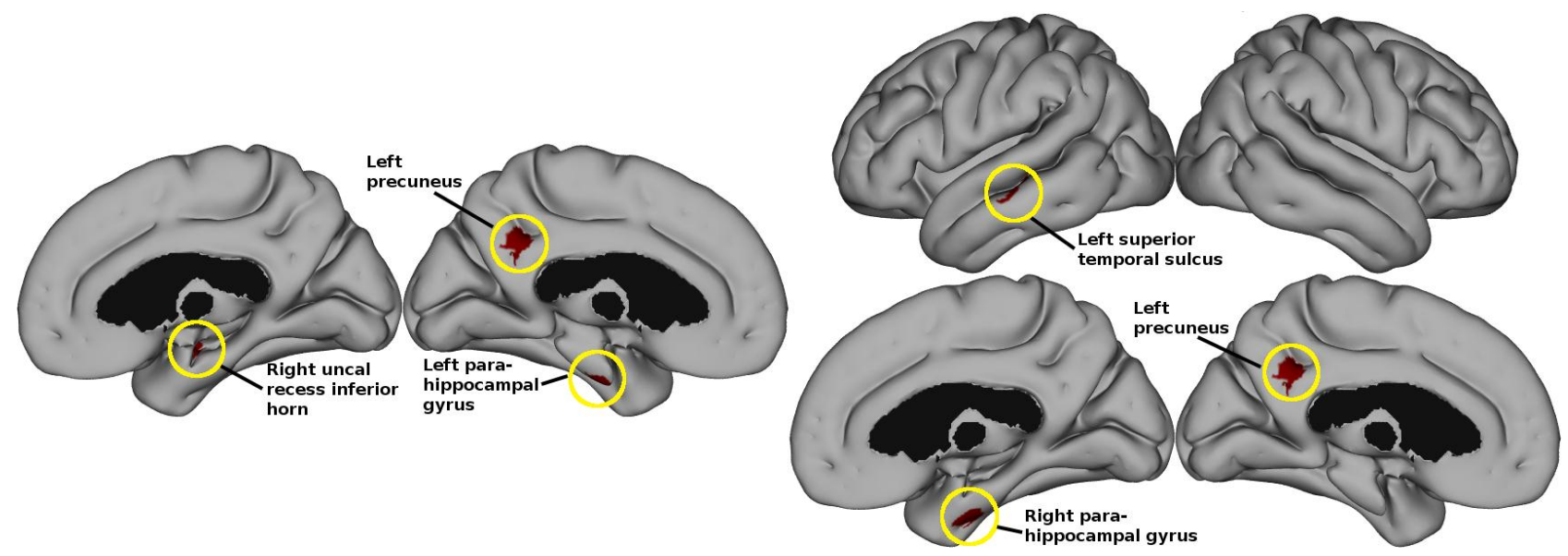

Figure 3. Thickness ROIs selected using thickness features only (left) and selected in the combined setup (right). Three ROIs are selected in both scenarios. In the combined setup, the ROls are moved away from the area around the HC. 

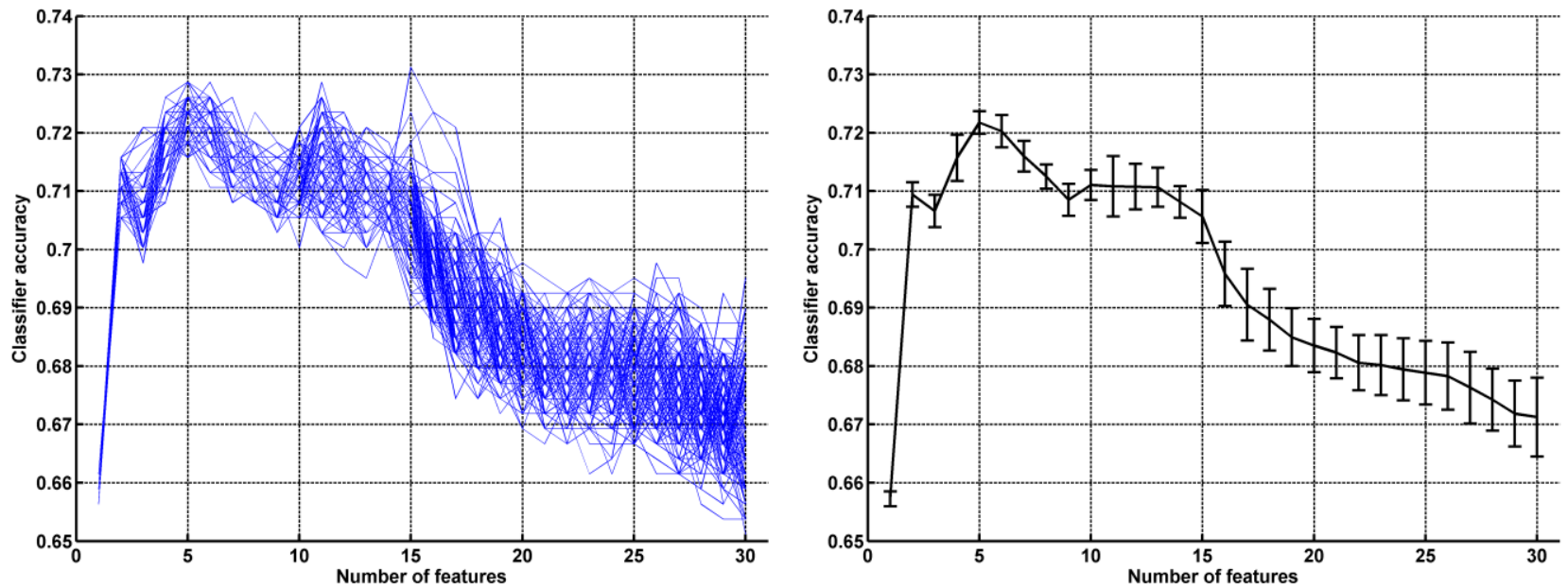

Figure 4. Accuracy of the LDA classifier as a function of number of selected features in the LOO experiments of the combined feature set. Left: curves for each LOO experiment. Right: mean curve showing standard errors. It can be observed that 5 features seem to be the optimal number for the classifier.
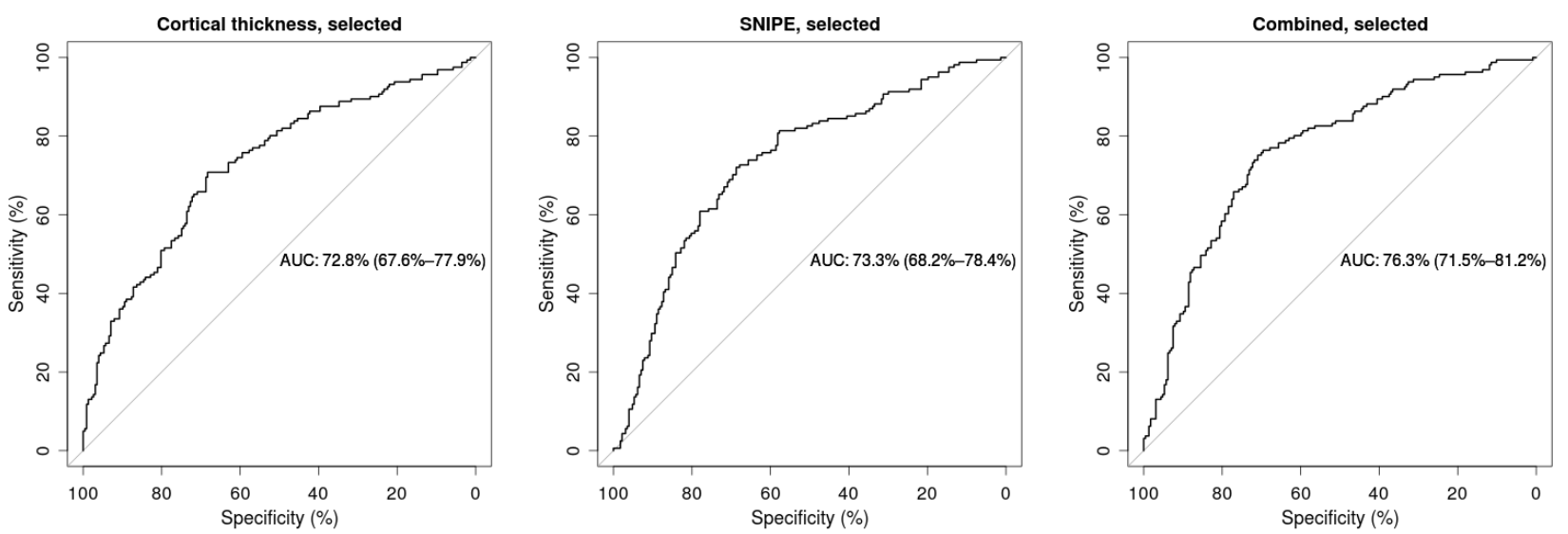

Figure 5. Receiver operating characteristic curves for the LDA classifier on the three different feature sets selected using mutual information features selection (MIFS). Area under the curve (AUC) is shown along with the $95 \%$ confidence interval computed with 2000 stratified bootstrap replicates (Robin et al., 2011). 